\title{
Interventions to Improve Menstrual Health in Low- and Middle-Income Countries: Do We Know What Works?
}

\author{
Julie Hennegan
}

\section{Menstruation Matters}

Menstrual health interventions have been motivated by growing recognition of the difficulties women face during menstruation, and the consequences of unmet menstrual needs. Qualitative studies have highlighted negative psychosocial outcomes associated with menstruation including distress, fear, shame, and anxiety, and have linked difficulties in managing menstrual bleeding with school absenteeism and disengagement (Sommer et al. 2015; Mason et al. 2013). Past quantitative studies on the links between menstrual management and school attendance have provided more mixed results (Grant, Lloyd, and Mensch 2013; Tegegne and Sisay 2014). However, more recent trials and cross-sectional studies have provided more support for this association (Montgomery et al. 2016; Miiro et al. 2018). Consistent with absences from school, there is self-reported evidence that girls avoid other activities such as physical exercise, work, or household tasks while menstruating (Hennegan et al. 2016a; Chandra-Mouli and Patel 2017). Furthermore, poor menstrual hygiene has been posited to lead to negative physical health outcomes including reproductive and urinary tract infections, with some emerging evidence of this association (Das et al. 2015; Phillips-Howard et al. 2016b). A study in Kenya has suggested that girls may be at risk of further harm through engaging in transactional sex to obtain menstrual supplies (Phillips-Howard et al. 2015).

Multiple actors have responded to reports of women's challenges with menstruation. A growing list of governments, international organizations, and NGOs have invested in menstrual health. Most of this attention has 
accelerated within the last decade, with many programs now advertising extensive reach. This sharp increase in the number of organizations seeking to intervene means increasing numbers of women and girls are involved in menstrual health interventions (Bobel 2019).

\section{How Do We Know What Works?}

While swift action to address unmet menstrual needs is laudable, good intentions do not guarantee positive outcomes. Seemingly intuitive community programs or policies often fail to have the anticipated results and can risk unintended harms (Hennegan 2017). Further, restricted budgets calling for a need to do more with less, place a premium on the identification and improvement of efficient interventions. For the objective evaluation of the effectiveness of an intervention, randomized controlled trials have long represented the gold standard (Gottfredson et al. 2015). While such studies are not sufficient to fully understand a program, and other complementary methods such as in-depth interviewing to capture rich qualitative data on experiences and contextual influences are needed (Gambrill 2006; Lewin, Glenton, and Oxman 2009), high-quality trials are able to minimize the many biases inherent in other designs. By collating multiple trials, systematic reviews can draw together this evidence to assess 'what works.' Systematic reviews apply a structured methodology to appraise, critique, and synthesize extant findings. They can estimate the pooled effect of interventions and can identify gaps in current research, methodological weaknesses, and provide guidance for future improvements.

This chapter draws on the findings of a systematic review which aimed to collate and appraise trials of the effectiveness of menstrual health interventions for improving education and psychosocial outcomes in low- and middle-income countries (Hennegan and Montgomery 2016). The review did not include studies exclusively assessing impacts on reproductive tract infection outcomes as another systematic review had focused on this issue (Sumpter and Torondel 2013). Study designs eligible for inclusion in the review included randomized and cluster randomized controlled trials, as well as non-randomized controlled trials including more than one intervention and control site. The review undertook a systematic search of 15 databases, as well as handsearching, grey literature search, ${ }^{1}$ checks of reference lists, and contact with experts. Searches were undertaken in January 2015. For this chapter, database searches, and citation tracking of papers included in the review were undertaken in December 2017 to identify additional eligible studies published between 2015 and 2017. The original review identified a total of eight eligible trials, with a further four trials published since the initial search. Thus, this chapter evaluates a total of 12 trials.

The subsequent sections of this chapter will summarize menstrual health interventions that have been trialed to date and provide insights into their 
effectiveness. It will reflect on what we do, and more often do not, know about what works in meeting the menstrual needs of women and girls in lowand middle-income settings.

\section{INTERVENTIONS}

Menstrual health is multifaceted. Hygienic menstrual practices and positive experiences of menstruation are dependent on the physical and social environment. Women and girls report needing knowledge and education about menstruation to understand their bodies, inform effective and hygienic practices, and to dispel unhelpful myths or taboos (Chothe et al. 2014). They also need clean materials to absorb or catch menses, as well as infrastructure to support safe locations for changing absorbents, and access to water, soap, and locations for washing reusable materials and their bodies (Sommer and Sahin 2013). Menstruation occurs in a context of social norms that may positively or negatively influence experience and dictate behavior. Further, the presence or lack of support from key individuals such as parents, teachers, and friends shapes experiences (Geertz et al. 2016; Hennegan et al. 2017). Menstrual needs are interconnected, and women and girls may face deprivations across several contributing factors. For the purposes of categorizing interventions, there has been a distinction between the types of interventions that provide a physical resource such as a menstrual absorbent or water, sanitation and hygiene (WASH) facilities, and programs providing psychosocial intervention which may include education, or efforts to address harmful taboos and stigma (Sumpter and Torondel 2013; Hennegan and Montgomery 2016; Geertz et al. 2016). The latter aimed at addressing psychosocial deprivations have been termed software interventions, while the former targeted at material deprivations have been termed hardware interventions. These interventions may include multiple components, and software and hardware interventions may be combined.

\section{Software Interventions}

A lack of menstrual knowledge has been identified across countries as a challenge to positive menstrual experiences (Chandra-Mouli and Patel 2017). Adolescent girls, particularly around the time of menarche, have been found to lack information on the function and origin of menstruation (Crichton et al. 2013). Many girls report being unaware that menstruation would occur, contributing to distress and fear at onset (Chandra-Mouli and Patel 2017). A lack of understanding of menstruation and strategies for hygienic menstrual practices are likely to perpetuate distress over subsequent menses and may contribute to negative effects on health and education. Deficits in knowledge are often combined with misinformation evoking further fear. Studies have reported taboos around the disposal of menstrual blood 
or consequences of others seeing used menstrual materials (Sommer et al. 2015), others have documented cultural expectations which explicitly restrict participation in social settings, bathing, cooking, or religious practices while menstruating (Chandra-Mouli and Patel 2017). At the same time, the stigmatized nature of menstruation across settings results in more implicit behavioral norms such as the expectation to keep menstrual status hidden and secret at all times (Jewitt and Ryley 2014). This prevents women and girls from discussing menstruation openly and receiving advice, support, or direction to health services where abnormalities are present (McMahon et al. 2011). Silence around menstrual issues may also mean that these needs are not prioritized in household budgets and girls are not equipped with the resources to assist the management of menstrual bleeding. Men and boys similarly lack knowledge and accurate information about menstruation and may perpetuate negative attitudes (Mason et al. 2017).

Software interventions include all interventions designed to address these highlighted psychosocial deprivations. Five trials of software interventions were included in the 2016 systematic review, with a subsequent three trials with an independent software condition published since this time. Greater detail regarding the design of these studies and risk of bias assessments are available in the original review (see Hennegan and Montgomery 2016). Software interventions to date have focused on the provision of menstrual education via a range of modalities, including; written materials such as leaflets and posters (Mbizvo et al. 1997), school-based education sessions provided by social workers ranging from one 120-minute session (Fetohy 2007 ) to ten 2 hour sessions (Fakhri et al. 2012), and peer, school, or health provider-led education approaches (Djalalinia et al. 2012; Abedian et al. 2011). An additional trial undertaken in Iran, published in 2017 (Afsari et al. 2017), compared the effectiveness of educating mothers to that of educating girls. Limited information was provided in these studies on the contents of the education offered. Most included statements that education included information on the definition and purpose of menstruation, the cyclic nature and timing, and other physical changes associated with puberty. Broad statements about education on 'self-care' and management practices were included with limited detail. There was a high risk of bias across these studies, with deficits in reporting, preregistration, and issues with participant attrition (dropout) and inappropriate treatment of outcome variables.

A more recent cluster-randomized trial provided a pilot evaluation of the Growth and Changes puberty education book in Ethiopia, based on qualitative research in multiple countries (Blake et al. 2017). This study identified improvements in knowledge about menstruation and decreases in fear and shame associated with menstruation at four-week follow-up. The trial used a clustered design including 20 schools. However, intervention and control conditions were assigned at the district, rather than school level. As such, the study only allocated one intervention and one control condition and results 
should be interpreted with caution. Qualitative components of the study triangulated quantitative findings with girls reporting the books reduced shame and confusion and helped to overcome secrecy around menstruation (Blake et al. 2017).

One additional pilot trial in Ghana included in the 2016 systematic review (Hennegan and Montgomery 2016) included both hardware and software conditions, with an education-alone condition allowing for a comparison of the effectiveness to a control. This study was the only one to assess impacts of education on school attendance rather than knowledge outcomes, however it was a small pilot with one education-alone and one control site (Montgomery et al. 2012). The study observed improvements in school attendance at 5-month follow-up. This Ghana pilot was extended to a larger study of eight schools in Uganda, two of which received an education-only intervention (Montgomery et al. 2016). The larger trial replicated the positive effects on school attendance. Qualitative follow-up of the trial identified avenues for improvement in the education provided (Hennegan et al. 2017). This study found that, beyond providing an awareness of menstruation prior to menarche, girls reported the practical information around managing menstrual bleeding to be particularly useful, and that more was desired. In interviews at the conclusion of the study, girls felt they still lacked information about vaginal discharge, menstrual irregularities, and reproductive tract symptoms. The study also found that social support, likely resulting from a shared language and education around menstruation, was particularly important and a possible driver of the positive effects identified in the quantitative results. Support from teachers and peers dictated girls' menstrual experience at school and contributed to their comfort and confidence to manage menstruation (Hennegan et al. 2017).

Taken together, extant trials of software interventions indicate that education interventions improve menstrual knowledge. It is unclear if this translates to impacts on psychosocial outcomes or wellbeing as identified in qualitative research as most studies failed to assess these outcomes. Of those that did, two identified improvements (Blake et al. 2017; Montgomery et al. 2012) and one reported no difference although suffered significant limitations due to participant dropout (Montgomery et al. 2016). Less evidence is available to support impacts on school attendance, although one program of work including a pilot and larger study indicates some promising results (Montgomery et al. 2012, 2016). More recent studies highlight the value of including complementary qualitative work alongside trials in the evaluation of menstrual health interventions (Montgomery et al. 2016; Blake et al. 2017). Hennegan et al. (2017) suggested that two levels of menstrual education may be needed. The first, and what appears to have received more attention in trials to date, is to provide girls with information about the basics of menstruation; what it is, why it happens, when it happens, and that this is a healthy and natural process linked to reproduction. This information 
helps to dispel fear of illness and shame by emphasizing the healthy nature of menstrual bleeding. The second level of education, which appears less prominently in trials, is more detailed information about menstrual experience and practical guidance. This may include suggestions on how to record or "chart" menstrual timing and bodily symptoms that might indicate the onset of menstruation so that girls may be aware, normal and abnormal vaginal discharge, irritation and pain, pain management strategies, and advice on absorptive materials and their hygienic management. Such education may be well placed to support effective practice, the identification of abnormalities, and equip girls to feel more informed, confident, and in control of their menstrual care and their bodies.

\section{Hardware Interventions}

To effectively and hygienically manage menstruation, women and girls need access to sufficient quantities of clean, safe, comfortable materials fit for the purpose of collecting menses (Sommer and Sahin 2013). Materials take many forms, from commercial disposable or reusable sanitary pads, tampons, and menstrual cups, to homemade pads or cloth. Women and girls may use different strategies depending on available resources, needs, and personal and cultural preferences. In low- and middle-income contexts, some women and girls may struggle to access or purchase sufficient materials supporting their preferences. This may lead to the use of alternatives which are not fit for purpose and are experienced as uncomfortable or ineffective (Ellis et al. 2016). Beyond materials, women and girls need access to private, safe locations to change menstrual materials, to wash and dry materials if they are reusable, and to wash their body with soap and water (Sommer and Sahin 2013). As such, supportive infrastructure, and WASH facilities are an essential enabler. These facilities are often unavailable or inadequate to meet women's and girls' physical and psychological needs (Fisher 2006; Hennegan et al. 2018). Due to the stigmatized nature of menstruation, women and girls have emphasized the need for a private space where they feel safe and comfortable to undertake menstrual practices such as changing or washing materials (Budhathoki et al. 2018). Locations for drying reusable absorbents are also needed. Hygiene guidelines have recommended drying absorbents in the sun to benefit from the antimicrobial properties of UV light, however, the perceived need to conceal menstruation means many girls report drying absorbents under mattresses or in other, hidden locations that are unlikely to facilitate hygiene (House et al. 2012; Hennegan et al. 2016b). Disposal of single-use menstrual absorbents has been largely overlooked in research and intervention to date. Few studies have investigated disposal practices, even when implementing single-use product interventions. In absence of waste management strategies, used products may be thrown into fields and waterways, pit latrines, or sanitation systems where they may cause blockages or 
health risks (Geertz et al. 2016). In addition to these environmental impacts, disposal presents a challenge for women and girls. Cultural taboos around others seeing menstrual blood, or the burning of menstrual blood add strain on finding acceptable disposal options and may contribute to distress and avoidance of social settings during menstruation (Sommer et al. 2015).

Hardware interventions have been conceptualized as programs providing material resources such as menstrual materials, infrastructure, or disposal facilities. However, to date, only the provision of menstrual materials has been trialed. Three eligible trials of hardware interventions were identified through systematic review (Hennegan and Montgomery 2016), with a further two studies published by the end of 2017 (Montgomery et al. 2016; Phillips-Howard et al. 2016b). Of the total five trials, two focused on the effectiveness of providing menstrual cups, two reusable sanitary pads, and one on disposable sanitary pads.

In the first trial of hardware provision for improving girls' menstrual management and school attendance, Oster and Thornton (2011) provided girls in Nepal, and their mothers, with a menstrual cup and instructions for use. They found no improvement for the girls provided with cups, compared to those who were not, however, school attendance was high for both groups at baseline and its results likely reflect a ceiling effect. A second study of menstrual cups was undertaken in Kenya and published in 2016 (Phillips-Howard et al. 2016b). The largest trial to date, this study included girls from 30 primary schools randomized to one of three conditions; a control, the provision of disposable menstrual pads, or the provision of a menstrual cup. Education was provided in all conditions. The study did not find any significant difference between conditions on the risk of school dropout over the one year follow-up period and did not report differences in school attendance as this data was collected using calendars given to participants and absences were very rarely reported, thus not analyzed. The study did not assess psychosocial outcomes but focused on sexually transmitted and reproductive tract infections as secondary outcomes, finding lower risks for those receiving either menstrual product (Phillips-Howard et al. 2016b).

The Ghana pilot study including both hardware and software components provided disposable sanitary pads in hardware conditions and found a moderate improvement in school attendance at three- and five-month follow-ups (Montgomery et al. 2012). This was followed by a larger study of reusable sanitary pads in Uganda published in 2016 (Montgomery et al. 2016). This study included four arms: two schools receiving education as noted above, two control schools, two receiving reusable pads alone, and two receiving combined education and product provision. The larger trial replicated results of the pilot with significant benefits for school attendance identified over the 24-month period, with no differences in the effects of the three different intervention conditions. The trial included only a small number of clusters and suffered from significant attrition with girls transferring or dropping 
out of school, with the longest follow-up period of any menstrual health intervention to date. Attrition levels suggest specific effect estimates of the trial should be interpreted with caution, with imputed analyses suggesting a beneficial effect of the pads or education conditions between 5.2 and 24.5\% (Montgomery et al. 2016). Qualitative follow-up supported the positive quantitative results (Hennegan et al. 2017). As noted above, social support due to the increased visibility of menstruation in schools may have contributed to intervention effects across schools. Girls reported largely positive experiences of the reusable pads which they felt were more comfortable and reliable, reducing fears of garment soiling. Another pilot study of homemade reusable sanitary pads in Uganda revealed a moderate, although nonsignificant effect likely due to the small sample size and number of clusters (Wilson, Josephine, and Pitt 2014).

Taken together, these studies suggest that providing menstrual materials may improve girls' school attendance, but that more research is needed. Studies varied in the outcomes assessed, duration, products provided, and design weaknesses making it difficult to draw conclusions. In comparison to the software interventions, hardware interventions focused more heavily on school attendance outcomes, and often neglected to assess more proximal change such as menstrual experience and distress. This could be facilitated through complementary qualitative research, and mediation and moderation methods undertaken with quantitative data collected as part of trial baseline and follow-up surveys. Research mapping connections between menstrual management and schooling and refinement of attendance measure techniques may improve outcome assessment in future trials (Miiro et al. 2018).

\section{Do We KnOW What Works?}

Menstrual health programming is rapidly increasing in scale (Bobel 2019). At the same time, trials of the effectiveness of interventions are limited in number and beset with limitations. There is some emerging evidence of the effectiveness of education interventions for improving knowledge, and for product provision interventions to encourage greater school attendance. However, methodological weaknesses across the evidence base mean no strong conclusions can be drawn (Hennegan and Montgomery 2016). More rigorous research is needed to evaluate interventions to improve menstrual health. Future trials must improve upon the limitations of past work. This includes improved reporting of studies, the use of adequate sample sizes, and strategies to mitigate attrition. Greater breadth is needed in the outcomes included in trials, for example, the assessment of menstrual experience in addition to school attendance. Across the studies presented in this review, there were limitations to the types of outcomes assessed and the way they were measured. Complementary quantitative and qualitative studies alongside trials may unearth additional understanding of participant experiences and lessons 
for intervention improvement. Moreover, as complex interventions within complex settings, understanding how the different aspects of interventions act and interact with the study context is key. This process can be facilitated by explicit theory on the mechanisms at play in interventions, and targeted investigation of these pathways during evaluation (Moore et al. 2015). Further, understanding the interaction between mechanisms of impact and the study context is critical for examining whether we would expect intervention effects to replicate in another setting, or at scale. For example, one currently registered trial lists sexually transmitted infections as primary outcomes in a trial of a menstrual health intervention but has not yet started an explicit framework for testing the pathways through which improved menstrual experiences will impact sexually transmitted infection rates (Clinical Trials 2017). One mechanism may be through reduced engagement in transactional sex to obtain menstrual materials (Phillips-Howard et al. 2015). If this is the case, positive effects identified in the trial are unlikely to replicate in settings where transactional sex is less prevalent.

One series of studies included in the systematic review included both hardware and software conditions. In both the Ghana pilot (Montgomery et al. 2012) and the follow-up in Uganda reported in Montgomery et al. (2016), education interventions and product provision interventions showed equal improvements in school attendance by the end of the follow-up period. It is unclear if these interventions influenced attendance and menstrual experience through different pathways, and future work is needed to unpack the potential of these interventions in combination. Careful attention to evaluate different parts of interventions is needed in development, piloting, and trial evaluation (Craig et al. 2008). This might mean the combination of hardware (for example, menstrual product or infrastructure provision) and software (for example, education or social norm) interventions, and also attention to more nuanced details such as education delivery mechanisms (for example, group-based, health provider presence), and content must be paid. More thorough evaluations may be able to identify best practices and suggest minimal or standard intervention packages which could be disseminated more broadly in programming and policy. For example, the identification of core educational components as has been helpful for guidance on the conduct of comprehensive sexuality education (UNESCO 2015). There is some evidence from sexual education studies that greater effect sizes may be achieved when adapting established programs, compared to those that are homegrown' (Kirby, Laris, and Rolleri 2006; Fonner et al. 2014). Similarities to sexual education for menstrual education mean that identifying and core components of education, that can be adapted to local needs, could be valuable in improving effectiveness. This may also reduce the time needed to invest in development; an important consideration for resource-constrained organizations. 
Beyond improving methodology in the evaluation of menstrual health interventions, more research and funding for intervention development is needed. Many factors contributing to women's and girls' menstrual experiences have not yet been addressed by tested interventions. Current education interventions may help dispel myths and stigma around menstruation, however, there have been no trials of interventions designed to target this important barrier to menstrual health. The perceived need to conceal menstrual status to minimize embarrassment contributes to women's and girls' distress during menstruation, avoidance of social settings (including school), and heightens the need for private locations for menstrual management and restricts washing, drying, and disposal choices. More research is needed to unpack the potential for interventions targeting menstrual stigma and their influence on hygiene practices, help-seeking, and menstrual experience more broadly. Importantly, education about menstruation alone is unlikely to be sufficient to change social norms (Bicchieri 2017). Mass media and other community programs to normalize menstruation may have positive influences at a population or community level and provide more supportive environments for women and girls. Studies of the influence of mass-media campaigns have been undertaken for other health and hygiene challenges, such as water and sanitation products, diarrheal disease, immunization, nutrition, mother-to-child transmission of HIV, and reproductive health, and could inform studies of these approaches to menstrual health (Naugle and Hornik 2014; Evans et al. 2014). Interventions that target parental or male attitudes to menstruation have received little attention. One upcoming cluster RCT with multiple intervention arms will include a condition providing both school level and community level education (Sol et al. 2019) and may provide new insights on the effectiveness of this approach. No studies have trialed improvements to WASH or other infrastructure to support menstrual management. These infrastructure challenges are relevant in home, school, and work environments and interventions have the potential to be integrated into other campaigns such as those aiming to improve sanitation coverage (Hennegan et al. 2018).

New intervention approaches to address menstrual health are varied. They range from more detailed and comprehensive education on menstrual health and body literacy, to reducing stigma and taboo at all levels, and infrastructure and WASH facilities to support menstrual activities, as well as integrated packages of these components. Moreover, menstrual pain and disorders have been largely neglected in trials and cross-sectional studies and populations such as those in humanitarian settings, those living with disabilities, girls outside of schools, and adult women at home and in the workplace have received inadequate attention (Sommer, Chandraratna, et al. 2016).

To support the development and evaluation of such interventions an improved evidence base is needed. This should include funding and conduct of non-trial methods such as cross-sectional and longitudinal designs to 
inform the breadth of menstrual needs, women's and girls' experiences and preferences, and to build theory around the links between menstruation and target outcomes such as health, education, and wellbeing (Hennegan 2017; Phillips-Howard et al. 2016a). This would inform intervention design as well as outcome assessment in trials. Greater integration of NGO, multinational programming, and academic research efforts would be of great value. NGO programs often only include case-study assessments of effectiveness but have often described more integrated approaches to intervention development (Sommer, Sahin, et al. 2016). Sufficient funding must be allocated to evaluation, to ensure that policies and programs are evidence-based and effective prior to large-scale dissemination. In the absence of evidence for the effectiveness of interventions, implementing organizations and advocates should proceed with caution. Integrated monitoring strategies should pay special attention to unintended harms. With considerable attention to methodological challenges, studies can improve understanding of menstrual health and develop and evaluate interventions which are well placed to improve women's and girls' menstrual health.

\section{Note}

1. Handsearching refers to manual searches of the table of contents of relevant journals or conference proceedings. Grey literature searching includes searches on websites and databases for 'grey literature,' that is, research that has been reported outside of peer-reviewed publications.

\section{REFERENCES}

Abedian, Zahra, Maryam Kabirian, Seyed Reza Mazlom, and Behroz Mahram. 2011. "The Effects of Peer Education on Health Behaviors in Girls with Dysmenorrhea." Journal of American Science 7 (1): 431-38.

Afsari, Atousa, Mojgan Mirghafourvand, Sousan Valizadeh, Massomeh Abbasnezhadeh, Mina Galshi, and Samira Fatahi. 2017. "The Effects of Educating Mothers and Girls on the Girls' Attitudes Toward Puberty Health: A Randomized Controlled Trial." International Journal of Adolescent Medicine and Health 29 (2): 984.

Bicchieri, Cristina. 2017. Norms in the Wild: How to Diagnose, Measure, and Change Social Norms. New York: Oxford University Press.

Blake, Sarah, Melissa Boone, Alene Yenew Kassa, and Marni Sommer. 2017. "Teaching Girls about Puberty and Menstrual Hygiene Management in Rural Ethiopia: Findings from a Pilot Evaluation." Journal of Adolescent Research 33 (5): 623-46. https://doi.org/10.1177/0743558417701246.

Bobel, Chris. 2019. The Managed Body. Cham: Palgrave Macmillan.

Budhathoki, Shyam Sundar, Meika Bhattachan, Enrique Castro-Sánchez, Reshu Agrawal Sagtani, Rajan Bikram Rayamajhi, Pramila Rai, and Gaurav Sharma. 2018. "Menstrual Hygiene Management among Women and Adolescent Girls in the Aftermath of the Earthquake in Nepal." BMC Women's Health 18 (1): 33. 
Chandra-Mouli, Venkatraman, and Sheila Vipul Patel. 2017. "Mapping the Knowledge and Understanding of Menarche, Menstrual Hygiene and Menstrual Health among Adolescent Girls in Low-and Middle-Income Countries.” Reproductive Health 14 (1): 30.

Chothe, Vikas, Jagdish Khubchandani, Denise Seabert, Mahesh Asalkar, Sarika Rakshe, Arti Firke, Inuka Midha, and Robert Simmons. 2014. "Students' Perceptions and Doubts about Menstruation in Developing Countries a Case Study from India." Health Promotion Practice 15 (3): 319-26. https://doi.org/ $10.1177 / 1524839914525175$.

Clinical Trials. 2017. "Cups or Cash for Girls Trial to Reduce Sexual and Reproductive Harm and School Dropout (CCg)." ClinicalTrials.gov Identifier: NCT03051789. Retrieved from: https://clinicaltrials.gov/ct2/show/record/ NCT03051789?term=NCT03051789\&rank=l [Accessed March 2017].

Craig, Peter, Paul Dieppe, Sally Macintyre, Susan Michie, Irwin Nazareth, and Mark Petticrew. 2008. "Developing and Evaluating Complex Interventions: The New Medical Research Council Guidance.” BMJ 337: al655.

Crichton, Joanna, Jerry Okal, Caroline W. Kabiru, and Eliya Msiyaphazi Zulu. 2013. "Emotional and Psychosocial Aspects of Menstrual Poverty in Resource-Poor Settings: A Qualitative Study of the Experiences of Adolescent Girls in an Informal Settlement in Nairobi." Health Care for Women International 34 (10): 891-916.

Das, Padma, Kelly K. Baker, Ambarish Dutta, Tapoja Swain, Sunita Sahoo, Bhabani Sankar Das, Bijay Panda, Arati Nayak, Mary Bara, and Bibiana Bilung. 2015. "Menstrual Hygiene Practices, WASH Access and the Risk of Urogenital Infection in Women from Odisha, India." PLoS One 10 (6): e0130777.

Djalalinia, Shirin, Fahimeh Ramezani Tehrani, Hossein Malek Afzali, Farzaneh Hejazi, and Niloofar Peykari. 2012. "Parents or School Health Trainers, Which of Them Is Appropriate for Menstrual Health Education?" International Journal of Preventive Medicine 3 (9): 622.

Ellis, A., J. Haver, J. Villasenor, A. Parawan, M. Venkatesh, M. C. Freeman, and B. A. Caruso. 2016. "WASH Challenges to Girls' Menstrual Hygiene Management in Metro Manila, Masbate, and South Central Mindanao, Philippines." Waterlines 35 (3): 306-23.

Evans, William Douglas, S. K. Pattanayak, S. Young, J. Buszin, S. Rai, and Jasmine Wallace Bihm. 2014. "Social Marketing of Water and Sanitation Products: A Systematic Review of Peer-Reviewed Literature." Social Science \& Medicine 110: $18-25$.

Fakhri, Moloud, Zeinab Hamzehgardeshi, Nayereh A. Hajikhani Golchin, and Abdulhay Komili. 2012. "Promoting Menstrual Health among Persian Adolescent Girls from Low Socioeconomic Backgrounds: A Quasi-Experimental Study.” BMC Public Health 12 (1): 193.

Fetohy, Ebtisam M. 2007. "Impact of a Health Education Program for Secondary School Saudi Girls about Menstruation at Riyadh City." Journal of the Egyptian Public Health Association 82 (1-2): 105-26.

Fisher, Julie. 2006. "For Her It's the Big Issue: Putting Women at the Centre of Water Supply, Sanitation and Hygiene.” Available from: https://www.wsscc.org/ resources-feed/big-issue-putting-women-centre-water-supply-sanitation-hygiene/ [Accessed June 2016]. 
Fonner, Virginia A., Kevin S. Armstrong, Caitlin E. Kennedy, Kevin R. O'Reilly, and Michael D. Sweat. 2014. "School Based Sex Education and HIV Prevention in Low-and Middle-Income Countries: A Systematic Review and Meta-Analysis." PLoS One 9 (3): e89692.

Gambrill, Eileen. 2006. "Evidence-Based Practice and Policy: Choices Ahead." Research on Social Work Practice 16 (3): 338-57.

Geertz, Alexandra, Lakshmi Iyer, Perri Kasen, Francesca Mazzola, and Kyle Peterson. 2016. "An Opportunity to Address Menstrual Health and Gender Equity." FSG. Online. Available from: https://www.fsg.org/publications/opportunity-address-menstrual-health-and-gender-equity [Accessed January 2017].

Gottfredson, Denise C., Thomas D. Cook, Frances E. M. Gardner, Deborah Gorman-Smith, George W. Howe, Irwin N. Sandler, and Kathryn M. Zafft. 2015. "Standards of Evidence for Efficacy, Effectiveness, and Scale-Up Research in Prevention Science: Next Generation.” Prevention Science 16 (7): 893-926.

Grant, Monica, Cynthia Lloyd, and Barbara Mensch. 2013. "Menstruation and School Absenteeism: Evidence from Rural Malawi." Comparative Education Review 57 (2): 260-84.

Hennegan, J. 2017. “Menstrual Hygiene Management and Human Rights: The Case for an Evidence-Based Approach.” Women's Reproductive Health 4 (3): 212-31.

Hennegan, J., Catherine Dolan, Laurel Steinfield, and Paul Montgomery. 2017. "A Qualitative Understanding of the Effects of Reusable Sanitary Pads and Puberty Education: Implications for Future Research and Practice." Reproductive Health 14 (1): 78 .

Hennegan, Julie, Catherine Dolan, Maryalice Wu, Linda Scott, and Paul Montgomery. 2016a. "Schoolgirls' Experience and Appraisal of Menstrual Absorbents in Rural Uganda: A Cross-Sectional Evaluation of Reusable Sanitary Pads." Reproductive Health 13 (1): 143.

- 2016b. "Measuring the Prevalence and Impact of Poor Menstrual Hygiene Management: A Quantitative Survey of Schoolgirls in Rural Uganda." BMJ Open 6 (12): e 012596.

Hennegan, Julie, Linnea Zimmerman, Alexandra K. Shannon, Natalie G. Exum, Funmilola OlaOlorun, Elizabeth Omoluabi, and Kellogg J. Schwab. 2018. "The Relationship between Household Sanitation and Women's Experience of Menstrual Hygiene: Findings from a Cross-Sectional Survey in Kaduna State, Nigeria.” International Journal of Environmental Research and Public Health 15 (5): 905.

Hennegan, J., and Paul Montgomery. 2016. “Do Menstrual Hygiene Management Interventions Improve Education and Psychosocial Outcomes for Women and Girls in Low and Middle Income Countries? A Systematic Review." PLoS One 11 (2): e0146985.

House, Sarah, Sue Cavill, and Thérèse Mahon. 2012. Menstrual Hygiene Matters: A Resource for Improving Menstrual Hygiene around the World. WaterAid.

Jewitt, Sarah, and Harriet Ryley. 2014. “It's a Girl Thing: Menstruation, School Attendance, Spatial Mobility and Wider Gender Inequalities in Kenya." Geoforum 56: $137-47$.

Kirby, Douglas, B. A. Laris, and Lori Rolleri. 2006. "The Impact of Sex and HIV Education Programs in Schools and Communities on Sexual Behaviors among Young Adults.” Family Health International, Research Triangle Park, N.C., 17. 
Lewin, Simon, Claire Glenton, and Andrew D. Oxman. 2009. "Use of Qualitative Methods alongside Randomised Controlled Trials of Complex Healthcare Interventions: Methodological Study." BMJ 339: b3496.

Mason, L., E. Nyothach, K. Alexander, F. O. Odhiambo, A. Eleveld, J. Vulule, R. Rheingans, K. F. Laserson, A. Mohammed, and P. A. Phillips-Howard. 2013. "We Keep It Secret so No One Should Kno'-A Qualitative Study to Explore Young Schoolgirls Attitudes and Experiences with Menstruation in Rural Western Kenya.” PLoS One 8 (11): e79132.

Mason, Linda, Muthusamy Sivakami, Harshad Thakur, Narendra Kakade, Ashley Beauman, Kelly T. Alexander, Anna Maria van Eijke, Kayla F. Laserson, Mamita B. Thakkar, and Penelope A. Phillips-Howard. 2017. "We Do Not Know': A Qualitative Study Exploring Boys Perceptions of Menstruation in India." Reproductive Health 14 (1): 174.

Mbizvo, M. T., J. Kasule, V. Gupta, S. Rusakaniko, S. N. Kinoti, W. Mpanju-Shumbushu, A. J. Sebina-Zziwa, R. Mwateba, and J. Padayachy. 1997. "Effects of a Randomized Health Education Intervention on Aspects of Reproductive Health Knowledge and Reported Behaviour among Adolescents in Zimbabwe." Social Science \& Medicine 44 (5): 573-77.

McMahon, Shannon A., Peter J. Winch, Bethany A. Caruso, Alfredo F. Obure, Emily A. Ogutu, Imelda A. Ochari, and Richard D. Rheingans. 2011. "The Girl with Her Period Is the One to Hang Her Head' Reflections on Menstrual Management among Schoolgirls in Rural Kenya." BMC International Health and Human Rights $11(1): 7$.

Miiro, George, Rwamahe Rutakumwa, Jessica Nakiyingi-Miiro, Kevin Nakuya, Saidat Musoke, Juliet Namakula, Suzanna Francis, Belen Torondel, Lorna J. Gibson, and David A. Ross. 2018. "Menstrual Health and School Absenteeism among Adolescent Girls in Uganda (MENISCUS): A Feasibility Study." BMC Women's Health 18 (1): 4.

Montgomery, Paul, Caitlin R. Ryus, Catherine S. Dolan, Sue Dopson, and Linda M. Scott. 2012. "Sanitary Pad Interventions for Girls' Education in Ghana: A Pilot Study." PLoS One 7 (10): e48274.

Montgomery, Paul, Julie Hennegan, Catherine Dolan, Maryalice Wu, Laurel Steinfield, and Linda Scott. 2016. "Menstruation and the Cycle of Poverty: A Cluster Quasi-Randomised Control Trial of Sanitary Pad and Puberty Education Provision in Uganda." PLoS One 11 (12): e0166122-e0166122.

Moore, Graham F., Suzanne Audrey, Mary Barker, Lyndal Bond, Chris Bonell, Wendy Hardeman, Laurence Moore, et al. 2015. "Process Evaluation of Complex Interventions: Medical Research Council Guidance." BMJ 350 (1): hl 258.

Naugle, Danielle A., and Robert C. Hornik. 2014. "Systematic Review of the Effectiveness of Mass Media Interventions for Child Survival in Low-and Middle-Income Countries." Journal of Health Communication 19 (supl): 190-215.

Oster, Emily, and Rebecca Thornton. 2011. "Menstruation, Sanitary Products, and School Attendance: Evidence from a Randomized Evaluation." American Economic Journal: Applied Economics 3 (1): 91-100. 
Phillips-Howard, Penelope A., Bethany Caruso, Belen Torondel, Garazi Zulaika, Murat Sahin, and Marni Sommer. 2016a. "Menstrual Hygiene Management among Adolescent Schoolgirls in Low-and Middle-Income Countries: Research Priorities." Global Health Action 9 (1): 33032.

Phillips-Howard, Penelope A., Elizabeth Nyothach, Feiko O. Ter Kuile, Jackton Omoto, Duolao Wang, Clement Zeh, Clayton Onyango, et al. 2016b. "Menstrual Cups and Sanitary Pads to Reduce School Attrition, and Sexually Transmitted and Reproductive Tract Infections: A Cluster Randomised Controlled Feasibility Study in Rural Western Kenya." BMJ Open 6 (11): e013229-e013229.

Phillips-Howard, Penelope A., George Otieno, Barbara Burmen, Frederick Otieno, Frederick Odongo, Clifford Odour, Elizabeth Nyothach, Nyanguara Amek, Emily Zielinski-Gutierrez, and Frank Odhiambo. 2015. "Menstrual Needs and Associations with Sexual and Reproductive Risks in Rural Kenyan Females: A Cross-Sectional Behavioral Survey Linked with HIV Prevalence." Journal of Women's Health 24 (10): 801-11.

Sol, Lidwien, V. Schölmerich, K. Liket, H. Alberda. 2019. The Ritu Study Protocol: A Cluster Randomized Controlled Trial of the Impact of Menstrual Health Programs on School Attendance and Wellbeing of Girls in Rural Bangladesh. Simavi: Amsterdam. Available from: https://simavi.org/what-we-do/programmes/ritu/ [Accessed January 2019].

Sommer, M., and Murat Sahin. 2013. "Overcoming the Taboo: Advancing the Global Agenda for Menstrual Hygiene Management for Schoolgirls." American Journal of Public Health 103 (9): 1556-59.

Sommer, M., M. Sahin, L. Paloubis, M. Troung, and J. Sinden. 2016. WASH in Schools Empowers Girls' Education: Proceedings of the Menstrual Hygiene Management in Schools Virtual Conference 2015. New York: United Nations Children's Fund and Columbia University.

Sommer, M., Nana Ackatia-Armah, Susan Connolly, and Dana Smiles. 2015. "A Comparison of the Menstruation and Education Experiences of Girls in Tanzania, Ghana, Cambodia and Ethiopia." Compare: A Journal of Comparative and International Education 45 (4): 589-609.

Sommer, M., S. Chandraratna, S. Cavill, T. Mahon, and P. A. Phillips-Howard. 2016. "Managing Menstruation in the Workplace: An Overlooked Issue in Low- and Middle Income Countries." International Journal for Equity in Health 15: 86.

Sumpter, Colin, and Belen Torondel. 2013. "A Systematic Review of the Health and Social Effects of Menstrual Hygiene Management.” PLoS One 8 (4): e62004.

Tegegne, Teketo Kassaw, and Mitike Molla Sisay. 2014. "Menstrual Hygiene Management and School Absenteeism among Female Adolescent Students in Northeast Ethiopia." BMC Public Health 14 (1): 1118.

UNESCO. 2015. Emerging Evidence, Lessons and Practice in Comprehensive Sexuality Education: A Global Review. Paris: United Nations Educational, Scientific and Cultural Organization.

Wilson, Emily, Josephine Reeve, and Alice Pitt. 2014. "Education. Period. Developing an Acceptable and Replicable Menstrual Hygiene Intervention.” Development in Practice 24 (1): 63-80. 
Open Access This chapter is licensed under the terms of the Creative Commons Attribution 4.0 International License (http://creativecommons.org/licenses/ by $/ 4.0 /)$, which permits use, sharing, adaptation, distribution and reproduction in any medium or format, as long as you give appropriate credit to the original author(s) and the source, provide a link to the Creative Commons license and indicate if changes were made.

The images or other third party material in this chapter are included in the chapter's Creative Commons license, unless indicated otherwise in a credit line to the material. If material is not included in the chapter's Creative Commons license and your intended use is not permitted by statutory regulation or exceeds the permitted use, you will need to obtain permission directly from the copyright holder. 